\title{
MANEJO DE LA CARCINOMATOSIS PERITONEAL DE ORIGEN COLORRECTAL Y REALIDAD EN PANAMÁ
}

\section{MANAGEMENT OF PERITONEAL CARCINOMATOSIS OF COLORECTAL ORIGIN AND REALITY IN PANAMA}

\author{
Candanedo, Olga*; Casiano, Gabriela*; Castillero, Isabella*; Cukier, Moisés ${ }^{\dagger}$ \\ *Estudiante del X Semestre de Medicina, Universidad de Panamá, Ciudad de Panamá \\ †Cirujano Oncólogo. Profesor de la Cátedra de Cirugía de la Facultad de Medicina. Universidad de Panamá
}

\author{
Recibido: 5 de mayo del 2020
}

Aceptado: 25 de mayo del 2020

Candanedo O, Casiano G, Castillero I, Cukier M. Manejo de la Carcinomatosis Peritoneal de Origen Colorrectal y Realidad en Panamá. Rev Méd Cient. 2020; 33(1):26-40. DOI: 10.37416/rmc.v33i1.568

\section{RESUMEN}

Antecedentes: En 1931, Sampson fue el primero en describir el término carcinomatosis peritoneal para referirse a la diseminación peritoneal de una neoplasia avanzada de ovario. Actualmente, engloba toda diseminación tumoral, masiva o regional, que involucra la serosa peritoneal y las estructuras anatómicas adyacentes. Cerca del $10 \%$ de los pacientes con carcinoma colorrectal presentan carcinomatosis peritoneal al momento del diagnóstico. Además, entre el $10 \%$ al $35 \%$ de los pacientes con recidiva presentarán lesiones exclusivamente a nivel peritoneal. En más de la mitad de estos pacientes el peritoneo es el primer y único sitio de metástasis.

Objetivo: Presentar la evidencia publicada sobre el abordaje y tratamiento quirúrgico de la carcinomatosis peritoneal de cáncer colorrectal en otras latitudes y como su implementación podría ofrecer un panorama prometedor para la calidad de vida de estos pacientes en Panamá.

Diseño: Se utilizaron artículos científicos indexados entre 2010 y 2020 que fueron seleccionados por su relevancia, validez y calidad de contenido; como aquellos disponibles en el: National Center for Biotechnology Information, Surgical Oncology Clinics of North America, Annals of Surgical Oncology, World Journal of Surgical Oncology; obtenidas a través de diversas bibliotecas virtuales.

Conclusión: La cirugía citorreductora en combinación con quimioterapia intraperitoneal hipertérmica ha mejorado la sobrevida global a los 5 años, de $40 \%$ a $51 \%$, dando resultados prometedores en pacientes con carcinomatosis peritoneal de origen colorrectal altamente seleccionados; e incluso se considera el único tratamiento potencialmente curativo.

Palabras Clave: carcinomatosis peritoneal, cáncer colorrectal, cirugía citorreductora, quimioterapia intraperitoneal hipertérmica.

\section{ABSTRACT}

Background: In 1931, Sampson was the first to describe the term peritoneal carcinomatosis to refer to the peritoneal spread of an advanced ovarian neoplasm. Today, it encompasses all tumor spread, massive or regional, involving the peritoneal serosa and adjacent anatomical structures. About $10 \%$ of patients with colorectal carcinoma present peritoneal carcinomatosis at the time of diagnosis. In addition, $10 \%$ to $35 \%$ of patients with recurrence will present lesions exclusively at the peritoneal level. In more than half of these patients the peritoneum is the first and only site of metastasis.

Objective: To present the published evidence on the approach and surgical treatment of peritoneal carcinomatosis of colorectal cancer in other latitudes and how its implementation could offer a promising panorama for the quality of life of these patients in Panama.

Design: We used scientific articles indexed between 2010 and 2020 that were selected for their relevance, validity and quality of content, such as those available at the National Center for Biotechnology Information, Surgical Oncology Clinics of North America, Annals of Surgical Oncology, World Journal of Surgical Oncology, obtained through various virtual libraries.

Conclusion: Cytoreductive surgery in combination with intraperitoneal hyperthermal chemotherapy has improved overall 5 -year survival from $40 \%$ to $51 \%$, giving promising results in patients with highly selected peritoneal carcinomatosis of colorectal origin; it is even considered the only potentially curative treatment.

Keywords: peritoneal carcinomatosis, colorectal cancer, cytoreductive surgery, hyperthermic intraperitoneal chemotherapy.

Manejo de la Carcinomatosis Peritoneal de Origen colorrectal y Realidad en Panamá by Candanedo, Casiano, Castillero, Cukier is licensed under a Creative Commons Attribution-NonCommercial-NoDerivs 4.0 Unported License. Permissions beyond the scope of this license may be available at www.revistamedicocientifica.org. 


\section{INTRODUCCIÓN}

En 1931, Sampson fue el primero en describir el término carcinomatosis peritoneal (CP) para referirse a la diseminación peritoneal de una neoplasia avanzada de ovario. Actualmente, engloba toda diseminación tumoral, masiva o regional, que involucra la serosa peritoneal y las estructuras anatómicas adyacentes. (1) Sucede por aquellas células tumorales que tienen la capacidad, en ciertos tipos de cáncer, de traspasar la membrana peritoneal y lograr la diseminación dentro de la misma, considerándose de pobre pronóstico con una sobrevida global de menos de seis meses después de su diagnóstico. ${ }^{(2,3)}$ Cerca del $10 \%$ de los pacientes con carcinoma colorrectal (CCR) presentan CP al momento del diagnóstico. Además, entre el $10 \%$ al 35\% de los pacientes con recidiva presentarán lesiones exclusivamente a nivel peritoneal. En más de la mitad de estos pacientes el peritoneo es el primer y único sitio de metástasis. En un $20 \%$ de los pacientes con CCR se dificulta ofrecer un manejo terapéutico por su asociación con CP. ${ }^{(1,4)}$ EI CCR presenta una tasa de incidencia de 7.3 por 100000 habitantes en Panamá, representando la segunda causa de muerte por cáncer, y con predominio principal en las provincias de Panamá, Chiriquí y Colón. ${ }^{(5)}$ Anteriormente, la CP era considerada como un escenario clínico sin opciones terapéuticas con intento curativo. La cirugía citorreductora (CRS) en combinación con quimioterapia intraperitoneal hipertérmica (HIPEC, del inglés, "hyperthermic intraperitoneal chemotherapy") ha mejorado la sobrevida global a los 5 años, de $40 \%$ a $51 \%$, dando resultados prometedores en pacientes altamente seleccionados e incluso se considera el único tratamiento potencialmente curativo. ${ }^{(2)}$

Nuestro objetivo con el siguiente trabajo es presentar la evidencia publicada sobre el abordaje y tratamiento quirúrgico de la carcinomatosis peritoneal de cáncer colorrectal (CPCCR) en otras latitudes y como su implementación podría ofrecer un panorama prometedor para la calidad de vida de estos pacientes en Panamá.

\section{GENERALIDADES}

La carcinomatosis peritoneal es la diseminación de células cancerígenas a través del revestimiento de la cavidad abdominal y ha sido considerada históricamente como una enfermedad diseminada, sistémica, incurable y con pronóstico ominoso a corto plazo independientemente de su origen. ${ }^{(6)}$ La siembra intraperitoneal por medio del líquido ascítico es una de las maneras más considerables de metástasis peritoneales y la principal causa de la CP. Al momento que las células cancerosas se difunden en la cavidad peritoneal se propagan a los diferentes sitios anatómicos del abdomen por medio de tres fuerzas básicas: gravedad, movimiento peristáltico y presión negativa ejercida por los movimientos de los músculos diafragmáticos. ${ }^{(7)}$ Puede presentarse de forma asintomática en sus primeras etapas o a medida que progresa la enfermedad, pueden manifestarse síntomas como náuseas, diarrea, distensión con dolor abdominal y pérdida de peso. La enfermedad se descubre a menudo cuando se evidencia ascitis u obstrucción intestinal, consecuente a una carga tumoral superior, complicando el tratamiento. ${ }^{(2)}$

En la actualidad, el diagnóstico preoperatorio de la CP resulta complicado. Si es detectado tardíamente, la CP suele dejar la enfermedad con opciones terapéuticas reducidas. ${ }^{(8)}$ Las imágenes anatómicas son el cimiento de la evaluación de las siembras peritoneales, pero implantes neoplásicos de menor tamaño pueden ser difíciles de detectar con tomografía computarizada (TC) o resonancia magnética. El 2-(Flúor 18) fluoro-2-desoxi-D-glucosa (FDG) Tomografía por Emisión de Positrones (PET, por sus siglas en inglés), está indicado en pacientes con marcadores tumorales elevados, con imágenes anatómicas negativas o inconclusas y en aquellos pacientes candidatos para CRS/HIPEC. ${ }^{(9)}$ En el estudio Franiel et $a l^{(10)}$ en 2009 , explican que la TC para el diagnóstico en pacientes con CP tiene sensibilidad entre $41 \%$ a $93 \%$ y especificidad entre $78 \%$ a 96\%. Entre las ventajas de los estudios de imágenes preoperatorias está el prevenir procedimientos invasivos como la laparoscopia o la laparotomía injustificada en los pacientes con enfermedades no resecables. ${ }^{(11)}$ El criterio de referencia para el diagnóstico de la CP sigue siendo la visualización peritoneal directa, permitiendo realizar el diagnóstico, establecer la localización y la extensión, además de permitir la recolección de biopsias para determinar el tipo histológico. ${ }^{(1)}$ Con el propósito de mejorar el diagnóstico temprano de estos pacientes, McMullen et al (8) plantearon la utilidad de la detección de biomarcadores liberados por la CP dentro de los biofluidos tanto en el suero como en el líquido ascítico. Esto proporciona una fuente probable de una modalidad de diagnóstico sensible y específica para detectar la CP en las etapas más tratables. ${ }^{(1)}$

\section{INDICACIONES}

En 1998 se realizó el primer estudio aleatorizado controlado de la CPCCR. Este ensayo asignó a los pacientes el tratamiento 
actual de ese entonces: quimioterapia sistémica con 5fluoracilo (5-FU) sola o CRS/HIPEC seguida de quimioterapia sistémica con 5-FU. El estudio evidenció que la sobrevida se duplicó en comparación con el grupo control (mediana de sobrevida de 22.3 meses frente a 12.6 meses). Por otro lado, los pacientes con afectación de seis o siete regiones abdominales estuvieron asociados a $80 \%$ de la toxicidad grado 4 y las muertes relacionadas con el tratamiento, razón por la cual estos pacientes no se beneficiaron de la citorreducción. No obstante, los pacientes con 0 a 5 regiones involucradas obtuvieron mejor beneficio, con una mediana de sobrevida mayor a 29 meses, a largo plazo de 48 meses y una tasa del $45 \%$ a los 5 años para aquellos pacientes que lograron tener una citorreducción completa. (12) Este estudio demostró que en pacientes cuidadosamente seleccionados se pueden minimizar la morbimortalidad, maximizar los beneficios y así obtener una mejor sobrevida a largo plazo.

La selección de los pacientes implica una evaluación profunda de los factores clínicos, patológicos y anatómicos. Baratti et al., estudiaron 426 pacientes sometidos a CRS/HIPEC que tenían como factores de riesgo: un índice de carcinomatosis peritoneal (ICP) superior a 30, más de cinco resecciones viscerales y puntuación de Eastern Cooperative Oncology Group (ECOG) superior a 0 , de tal manera que los pacientes con 2 factores de riesgo tuvieron una incidencia de morbilidad mayor del 65,7\% y un riesgo de mortalidad del $16,6 \%$. Los pacientes con los tres factores de riesgo tenían una morbilidad del $100 \%$ y una mortalidad del 22.2\%. ${ }^{(13)}$ Para el MD Anderson Cancer Center, los mejores candidatos para la CRS/HIPEC son pacientes menores de 70 años, no fumadores, con un estadío funcional de ECOG de 0 a 1, y en buen estado nutricional (albúmina >3.5 $\mathrm{g} / \mathrm{dL}$ ). Klaver et al (14) exponen en su estudio, que la CRS y la Quimioterapia intraperitoneal perioperatoria (PIC), en el cual se incluyeron veinticuatro pacientes con una edad media de 73.5 años, la mediana de sobrevida global fue de 35 meses con una tasa de sobrevida de 6, 12 y 18 meses de $94 \%$, 83\% y 68\%, respectivamente. Demostrando así, que la edad por sí sola no influye en los resultados de la cirugía para el CPCCR, y la sobrevida específica en estos pacientes es similar a la de los pacientes más jóvenes. La CRS/HIPEC generalmente están contraindicados en pacientes con una carga tumoral peritoneal extensa (ICP > 20), metástasis extraperitoneales concomitantes, comorbilidades graves y un estado de bajo rendimiento. ${ }^{(15)}$
El Grupo Internacional de Oncología de Superficie Peritoneal, elaboró una guía para la selección apropiada de pacientes con CPCCR en la que definieron ocho variables clínicas y radiológicas que aumentan la probabilidad de una citorreducción completa y, por lo tanto, mejoría en la sobrevida. ${ }^{(16)}$ (Ver tabla 1)

Tabla 1. Criterios de selección de pacientes para cirugía de citorreducción.

Estado funcional en la escala ECOG de 2 o menos.
No evidencia de enfermedad extra abdominal.
Hasta tres pequeñas metástasis hepáticas parenquimatosas
resecables.
No evidencia de obstrucción biliar.
No evidencia de obstrucción ureteral.
No evidencia de obstrucción intestinal en más de un sitio
Afectación del intestino delgado: en ausencia de enfermedad
grave en el mesenterio con varios sitios segmentados de
obstrucción parcial.
Enfermedad de pequeño volumen en ligamento gastrohepático

Fuente: Esquivel J, Sticca R, Sugarbaker P, Levine E, Yan TD, Alexander R, Baratti D, Bartlett D, Barone R, Barrios P, Bieligk S. Cytoreductive surgery and hyperthermic intraperitoneal chemotherapy in the management of peritoneal surface malignancies of colonic origin: a consensus statement. Annals of Surgical Oncology. 2007 Jan 1;14(1):128-33.(16)

En el gran estudio multiinstitucional realizado por Glehen et al,

(17) en el que se incluyó a 523 pacientes con CPCCR tratados con este enfoque terapéutico, se encontró una mediana de sobrevida global de 30 meses. Aunque los pacientes con metástasis extraperitoneales concomitantes generalmente no se consideran candidatos para CRS/HIPEC, los resultados de dicho estudio revelaron que incluso un pequeño número de pacientes con CPCCR con metástasis hepáticas concomitantes limitadas se sometieron a este procedimiento, especialmente desde 2008, con una mediana de sobrevida de 23 meses. Se realizó una resección en cuña no anatómica de las metástasis hepáticas en la gran mayoría de estos casos. El hecho de que un pequeño grupo de pacientes con $\mathrm{CP}$ con metástasis hepáticas concomitantes se sometieron a CRS/HIPEC resulta de la evidencia actual que indica que la afectación hepática no debe considerarse una contraindicación absoluta si las metástasis son confinadas a un máximo de tres lesiones bien definidas. Además, las tasas de complicaciones mayores y las tasas de mortalidad se mantuvieron comparables a un estudio reciente por Kuijpers et al., ${ }^{(18)}$ donde se evidenció en un $34 \%$ y $3 \%$ respectivamente, después de CRS y HIPEC. 
Por otro lado, el ICP es una herramienta útil en la evaluación intraoperatoria que relaciona la extensión de la enfermedad con la sobrevida. Sin embargo, utilizarla en la evaluación preoperatoria por medio de imágenes limita la evaluación de la extensión tumoral. En un estudio se compararon imágenes preoperatorias vs hallazgos quirúrgicos basándose en el ICP y demostraron que los índices se correlacionaban solo en el $74 \%$ de los pacientes. ${ }^{(19)}$ Otros estudios como el de Goéré et al. (20) demuestran que la CRS/HIPEC no ofrecen beneficio en pacientes con ICP $\geq 17$. Mientras que otros consideran puntos de corte más altos como ICP $\geq 20 .^{(21)}$ Recientemente, las recomendaciones multidisciplinarias del Consenso de Chicago para el tratamiento del CCR, específicamente en lo que se refiere al tratamiento de la malignidad de la superficie peritoneal, los pacientes con una puntuación ICP de 11-15 tuvieron una mejora en la sobrevida global con HIPEC (41,6 meses frente a 32,7 meses sólo para el CRS). ${ }^{(22)}$

Otro aspecto a tomar en cuenta es la histología, la presencia de células en anillo de sello es un marcador de mal pronóstico para estos pacientes. La Universidad de Pittsburgh refiere que la CRS/HIPEC no confiere un beneficio en aquellos pacientes que presentan una patología con células en anillo de sello a menos que se logre una citorreducción completa. ${ }^{(23)}$ Se han creado sistemas de puntuación que incorporan la histología y el ICP para que la selección sea más completa. La escala de gravedad de enfermedad de superficie peritoneal (PSDSS por sus siglas en inglés), la cual fue elaborada para clasificar a los pacientes al momento del diagnóstico de carcinomatosis, a fin de reducir al mínimo el exceso de tratamiento con una terapia multimodal agresiva, como la quimioterapia sistémica y la citorreducción. (Ver tabla 2). Esto se anotó como etapas I a IV basadas en la suma de las puntuaciones arbitrarias de los parámetros de estadificación PSDSS $1<4$, PSDSS $I I=4-7$, PSDSS $I I=$ = 8-10, y PSDSS IV $>10 .{ }^{(24)}$ El resultado de la sobrevida por etapas sugiere que incluso con una CRS/HIPEC, y una extensión moderada de la carcinomatosis, los pacientes con PSDSS en etapa IV obtienen un beneficio de sobrevida limitado, con una sobrevida global de aproximadamente 7 meses. ${ }^{(25)}$

Para mejorar aún más la selección, algunos autores han modificado la escala agregando el estado de mutación del gen RAS (RAS-PSDSS) proporcionando una evaluación más factible. ${ }^{(26)}$ De manera similar, se ha desarrollado el nomograma "The Colorectal Peritoneal Metastases Prognostic Surgical Score" del inglés (COMPASS), que abarca la edad, el ICP, el estado de los ganglios linfáticos N2 y la histología de las células en anillo de sello y predice la sobrevida a 1, 2 y 3 años en base a la puntuación compuesta (Ver figura 1).

Tabla 2. Puntuación de la gravedad de la enfermedad en la superficie del peritoneo.

\begin{tabular}{|c|c|c|}
\hline $\begin{array}{l}\text { Síntomas } \\
\text { clínicos* }\end{array}$ & $\begin{array}{l}\text { Grado de } \\
\text { carcinomatosis }\end{array}$ & Histopatología primaria \\
\hline $\begin{array}{l}\text { Sin síntomas } \\
\text { (0 puntos) }\end{array}$ & $\begin{array}{l}\text { ICP }<10 \\
\text { (1 punto) }\end{array}$ & $\begin{array}{l}\text { Bien o moderadamente } \\
\text { diferenciado y NO } \\
\text { (1 punto) }\end{array}$ \\
\hline $\begin{array}{l}\text { Síntomas leves } \\
\text { (1 punto) }\end{array}$ & $\begin{array}{l}\text { ICP } 10-20 \\
\text { (3 puntos) }\end{array}$ & $\begin{array}{l}\text { Moderadamente } \\
\text { diferenciado y N1 o N2 } \\
\text { (3 puntos) }\end{array}$ \\
\hline $\begin{array}{l}\text { Síntomas graves } \\
\text { (6 puntos) }\end{array}$ & $\begin{array}{l}\text { ICP }>20 \\
\text { (7 puntos) }\end{array}$ & $\begin{array}{l}\text { Células mal diferenciadas o } \\
\text { en anillo de sello } \\
\text { ( } 9 \text { puntos) }\end{array}$ \\
\hline
\end{tabular}

*Síntomas: leves (pérdida de peso $<10 \%$ del peso corporal, dolor abdominal leve, ascitis asintomática) o graves (pérdida de peso $>10 \%$ del peso corporal, dolor incesante, obstrucción intestinal, ascitis sintomática).

Fuente: Cashin, P. H., Graf, W., Nygren, P., \& Mahteme, H. (2013). Comparison of prognostic scores for patients with colorectal cancer peritoneal metastases treated with cytoreductive surgery and hyperthermic intraperitoneal chemotherapy. Annals of surgical oncology. ${ }^{(25)}$

Figura 1. Componentes del nomograma COMPASS

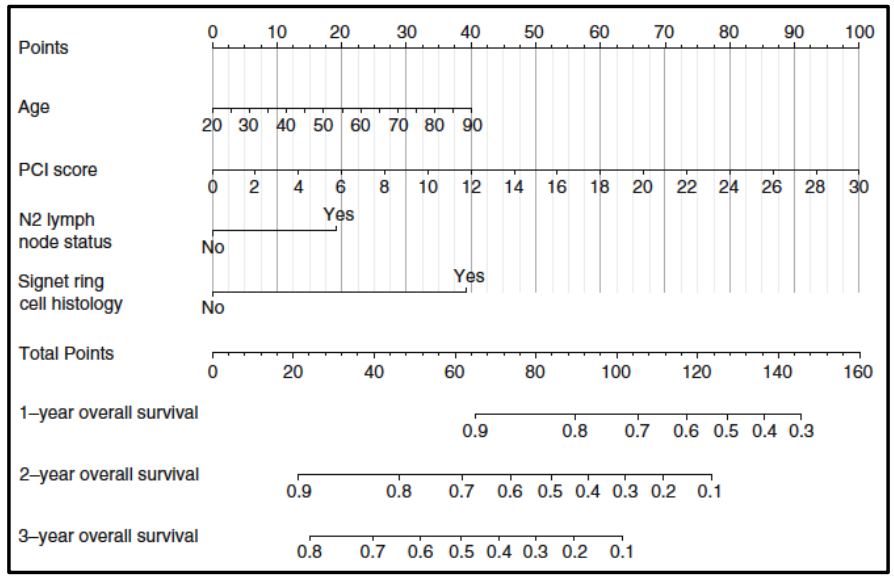

Fuente: Yong, Z.Z., Tan, G.H.C., Shannon, N. et al. P.R.O.P.S. - A novel Pre-Operative Predictive Score for unresectability in patients with colorectal peritoneal metastases being considered for cytoreductive surgery (CRS) and hyperthermic intraperitoneal chemotherapy (HIPEC). World J Surg Onc. (27)

Estas herramientas presentan la desventaja de incluir factores que necesitan ser evaluados dentro del quirófano limitando su uso en el entorno preoperatorio. Yong y colaboradores, realizaron un estudio retrospectivo con 56 pacientes con CPCCR, donde el objetivo era desarrollar una nueva Puntuación 
Pre-Operativa Predictiva (del inglés PROPS) que permitiera identificar aquellos pacientes que no fueran candidatos para el tratamiento. Esta puntuación incluye nueve factores que pueden ser identificados en la evaluación preoperatoria, clasificados en tres grandes grupos: pobre biología tumoral, fuerte carga tumoral y proliferación tumoral activa. El estudio demostró que PROPS tenía mayor capacidad de detectar casos irresecables (especificidad del 68\%) con una menor tasa de falsos positivos comparado con PSDSS. ${ }^{(27)}$

Además, se ha introducido la puntuación "Colorectal Peritoneal Score" del inglés (COREP) para la evaluación del pronóstico para la CRS/HIPEC, que consiste en una variable histopatológica, niveles de hemoglobina, recuento de glóbulos blancos, cuatro marcadores tumorales séricos (CEA, CA125, CA19-9, y CA15-3) y sus cambios entre la derivación y la cirugía. Esta refleja la biología del tumor y puede apoyar a la radiología para mejorar el proceso de selección de pacientes. El propósito es identificar a los pacientes con una sobrevida corta específica de la CP que puede ser inadecuada para el CRS y el HIPEC. Un puntaje COREP de 66 tiene una especificidad de $92-100 \%$ y una sensibilidad de $75-80 \%$ en la identificación de pacientes que sobrevivirán 12 meses. ${ }^{(24)}$

La puntuación COREP funciona mejor junto con la quimioterapia preoperatoria. ${ }^{(28)}$ De este modo, los pacientes que son referidos para el tratamiento de CRS/HIPEC de las CPCCR pueden ser sometidos a pruebas de valores de marcadores tumorales séricos antes y después de la quimioterapia neoadyuvante. Además, puede aplicarse después de 3 meses de terapia neoadyuvante; junto con estudios radiológicos, esto puede servir de base para una decisión de tratamiento: CRS/HIPEC, o continuar con la quimioterapia sistémica como tratamiento paliativo de primera línea; ${ }^{(24)}$ COREP ha sido útil para identificar a los pacientes que no se beneficiarán del tratamiento. ${ }^{(24,28)}$ La puntuación de la PSDSS se muestra como un indicador independiente de pronóstico en los pacientes con CPCCR que se someten a una completa CRS/HIPEC y puede aplicarse para adaptar el uso de tratamientos posteriores. ${ }^{(25)}$

\section{MANEJO}

Hasta hace poco, el tratamiento del paciente se limitaba a la cirugía paliativa y la quimioterapia sistémica, con tasas de sobrevida global de 4 a 12 meses. Durante la última década, han surgido nuevos descubrimientos sobre la diseminación tumoral intraperitoneal limitada, sin diseminación sistémica, lo que ha llevado a otros enfoques terapéuticos centrados en la cavidad abdominal y pélvica que pueden erradicar la CP y proporcionar una mayor sobrevida en personas estrictamente seleccionadas. $^{(29)}$ Hoy en día existe un tratamiento multidisciplinario para la CPCCR; este tratamiento consta de tres tipos de procedimientos distintos, pero que deben emplearse en conjunto para así obtener de ellos el resultado y beneficio esperado, estos son: la cirugía radical, quimioterapia intraperitoneal y la hipertermia.

\section{QUIMIOTERAPIA SISTÉMICA}

Luego de la cirugía, el tratamiento estándar de la CP de los tumores de colon ha sido la quimioterapia intravenosa, con una sobrevida global aproximada de unos 6 meses en la época del tratamiento con 5-FU-Leucovorin, pero no es hasta el año 2000 en el que surgen nuevos agentes citostáticos como el Irinotecan y el Oxaliplatino, que por medio de su asociación con el $5 \mathrm{FU}$ Leucovorin (FOLFOXIRI) consiguieron aumentar la sobrevida global en 22.6 meses. Posteriormente, con la consecuente aparición en 2004 de moléculas diana con capacidad de inhibición de los factores de crecimiento del endotelio vascular en forma de anticuerpo monoclonal Bevacizumab y su efecto antiangiogénico correspondiente, que en adición con FOLFOX (leucovorina/fluorouracilo/oxaliplatino) aumentó la sobrevida a 22.8 meses contribuyendo a un mayor rendimiento en el tratamiento de la CPCCR. ${ }^{(30)}$

En una reciente revisión sistemática Waite et al ${ }^{(31)}$ sugieren el efecto positivo de la quimioterapia adyuvante en la sobrevida global, a pesar de la heterogeneidad de los diferentes estudios. En este estudio, el número de ciclos de quimioterapia adyuvante no demostró una relación clara con la sobrevida; no obstante, uno de los estudio incluidos ${ }^{(32)}$ encontró una mejora significativa para los pacientes que se sometieron a seis o más ciclos en comparación con los que se sometieron a menos. ${ }^{(31)}$ De igual forma se adjuntó el trabajo por Chua et al, ${ }^{(33)} \mathrm{el} \mathrm{cual}$ comparó la sobrevida general de pacientes en relación con diferentes regímenes de quimioterapia sistémica, evidenciando que el uso de las terapias sistémicas modernas se asociaron con mejores resultados en pacientes con CPCCR tratados solo sistémicamente o con CRS/HIPEC. Este grupo dividió la terapia sistémica en quimioterapia estándar (5-fluorouracilo y leucovorina), quimioterapia moderna (5-fluorouracilo y leucovorina o capecitabina con oxaliplatino o irinotecán) y quimioterapia moderna con terapia biológica (quimioterapia moderna con bevacizumab, cetuximab, o panitumumab), concluyendo finalmente que el empleo de CRS/HIPE 
regímenes de quimioterapia moderna como modalidad combinada pueden lograr una sobrevida global del $40 \%$ a los 5 años en pacientes seleccionados.

Actualmente, el manejo multidisciplinario ha cambiado de la administración de quimioterapia adyuvante a la recomendación de quimioterapia preoperatoria o perioperatoria en los últimos años, en particular para pacientes con enfermedad N2 en el momento de la resección del tumor primario; tomando en cuenta que, si los pacientes ya habían recibido quimioterapia sistémica en los 2 años previos al diagnóstico de $\mathrm{CP}$, no se ofrecía quimioterapia sistémica antes o después de CRS/HIPEC. ${ }^{(34)}$ Sin embargo, un factor independiente imprescindible para una mejora en la sobrevida es la resección radical del tumor con intento curativo; por lo que, la efectividad de cualquier tratamiento está sujeta en gran medida a la extensión del tumor y la integridad de la citorreducción, procurando evitar un retraso en el tratamiento adyuvante por complicaciones quirúrgicas. ${ }^{(35)}$

La quimioterapia sistémica neoadyuvante consiste en un tratamiento que se administra como primer paso para reducir el tamaño del tumor antes de la cirugía. ${ }^{\left({ }^{30}\right)}$ La evidencia sobre que la quimioterapia neoadyuvante confiere beneficios de sobrevida en otros tumores, por ejemplo cáncer rectal, ${ }^{(36)}$ generó en Leimkühler et al (37) la incógnita sobre su posible eficacia mediante la utilización previa a CRS/HIPEC en pacientes con CCR, obteniendo resultados que mostraban que la quimioterapia neoadyuvante no influyó ni en la morbilidad ni en la tasa de mortalidad. Por su parte, Passot et al (38) publicaron el primer estudio que indicó la tasa de incidencia de respuesta patológica completa en pacientes con CPCCR después de la quimioterapia sistémica neoadyuvante; además, recalcaron el profundo impacto de este parámetro en el pronóstico de la sobrevida de los pacientes, alcanzando una tasa de respuesta completa del 9,7\%. De igual manera propusieron que, FOLFOX junto con bevacizumab conducen a una mejora en la sobrevida. Inclusive una revisión retrospectiva multi-institucional de pacientes con CPCCR sometidos a CRS con o sin HIPEC evidenció que aquellos pacientes que recibieron quimioterapia neoadyuvante experimentaron una sobrevida general más prolongada en comparación con los que se sometieron a cirugía primero; por lo que podemos deducir que, la quimioterapia neoadyuvante previo a la CRS con o sin HIPEC parece ser una técnica segura y viable para CPCCR, ayudando en la adecuada selección de pacientes para cirugía citorreductora agresiva. ${ }^{(39)}$

\section{CIRUGÍA RADICAL DE CITORREDUCCIÓN}

La CRS en la CP deriva de una estrategia quirúrgica descrita por Sugarbaker en 1985 al cual llamó procedimientos de peritonectomía. El objetivo es la erradicación del tumor macroscópico presente, además de las estructuras cercanas infiltradas por el mismo. En muchas ocasiones esto incluye la resección de órganos afectados (colon, estómago, bazo, recto), con el consiguiente contacto directo de los agentes de quimioterapia citotóxicos a altas temperaturas, para erradicar cualquier enfermedad residual. ${ }^{(2,40)}$

El pronóstico de los pacientes con $\mathrm{CP}$ que se someten a un tratamiento combinado se correlaciona con la carga tumoral medida como ICP y la capacidad de realizar un CRS, para eliminar completamente el tumor. El volumen de la enfermedad peritoneal debe determinarse tanto antes como después de la cirugía, ya que establece las indicaciones y las posibilidades de la CRS. ${ }^{(41)}$ Durante la fase inicial de la cirugía se evalúa la extensión de la enfermedad en la cavidad abdomino pélvica y se calcula el ICP descrito por Sugarbaker, que es una puntuación semicuantitativa que combina el tamaño de los nódulos tumorales con la distribución según 13 regiones abdominales (ver figura 2); obteniendo este índice que va de 0 a 39 puntos. Si el paciente tiene 20 puntos o menos, puede continuarse el plan de lograr una citorreducción óptima (ausencia de tumor visible), con uno o más de los siguientes procedimientos. ${ }^{(21)}$ (Ver tabla 3)

Para clasificar el grado de éxito en la CRS, se han propuesto varios sistemas para determinar el tamaño del tumor residual después de la cirugía. El método de clasificación más utilizado es el de "Completeness of Cytoreduction Score" (CC), el cual se basa en la capacidad de los agentes quimioterapéuticos administrados, por vía intraperitoneal, para penetrar en el tejido tumoral. ${ }^{(41)}$

Se asigna una puntuación de CC-0 cuando no se visualiza la siembra peritoneal dentro del campo operatorio. CC-1 indica nódulos peritoneales $<2,5 \mathrm{~mm}$ que persisten después de la citorreducción. CC-2 indica nódulos entre $2,5 \mathrm{~mm}$ y $2,5 \mathrm{~cm}$, mientras que la puntuación CC-3 indica nódulos $>2,5 \mathrm{~cm}$ en cualquier sitio dentro del abdomen o la pelvis. Aunque tanto el CC-0 como el CC-1 corresponden con una citorreducción completa, en la práctica clínica el CC-1 sólo se considera una medida apropiada de la eficacia del HIPEC en la CPCCR, mientras que el CC-0 se considera necesario antes de que se puedan alcanzar los máximos índices de sobrevida; CC-2 y CC-3 se designan como citorreducciones incompletas. ${ }^{(43)}$ 
Figura 2. Índice de carcinomatosis peritoneal (ICP).
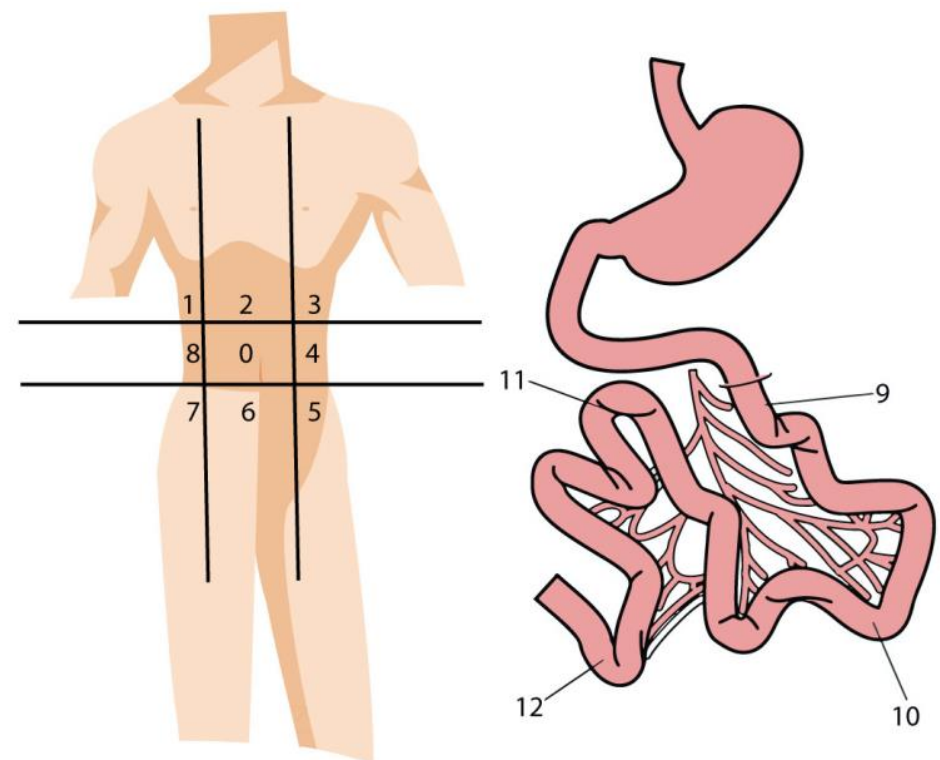

Regiones:

0 Central

1 Superior derecho

2 Epigastrio

3 Superior izquierdo

4 Flanco izquierdo

5 Inferior izquierdo

6 Pelvis

7 Inferior derecho

8 Yeyuno superior

9 Yeyuno inferior

10 Íleon superior

11 Íleoninferior
Puntaje del tamaño tumoral:

LS 0 No se observa tumor

LS 1 Tumor de hasta $0.5 \mathrm{~cm}$

LS 2 Tumor de hasta $5.0 \mathrm{~cm}$

LS 3 Tumor $>5.0 \mathrm{~cm}$ o confluencia

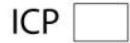

Dos planos transversales y dos planos sagitales dividen el abdomen en 9 regiones. El plano transversal superior se encuentra en el aspecto bajo el margen costal y el plano transversal inferior se sitúa en la espina ilíaca anterior superior. Los planos sagitales dividen el abdomen en 3 sectores iguales. Las líneas definen las 9 regiones enumeradas en el sentido de las agujas del reloj con el 0 en el ombligo y el 1 definiendo el espacio debajo del hemidiafragma derecho. Las regiones 9 a 12 dividen el intestino delgado. La puntuación del tamaño de la lesión se determina después de la lisis completa de todas las adherencias y la inspección completa de todas las superficies peritoneales parietales y viscerales.

Fuente: Granados García M, Arrieta Rodríguez O, de León C. Oncología y cirugía: bases y principios. 2013. ${ }^{(42)}$

Tabla 3. Procedimientos de citorreducción.

\section{Omentectomía extensa o mayor, peritonectomía parietal derecha +} resección de colon derecho.

Peritonectomía pélvica + resección de colon sigmoides + histerectomía con o sin ooforectomía.

Omentectomía extensa y disección del ligamento hepático + antrectomía + colecistectomía.

Peritonectomía de cuadrante superior derecho + cápsula de Glisson.

Peritonectomía de cuadrante superior derecho y esplenectomía.

Otra resección intestinal y/o resección de masa abdominal.

Fuente: López-Basave HN, Morales-Vásquez F, Luna Ortiz K, Méndez Herrera C, Ruiz-Molina JM. Citorreducción e HIPEC en carcinomatosis peritoneal. Experiencia del Instituto Nacional de Cancerología de México. Cirujano general. 2014;36(3):138-44. (21)

Se asigna una puntuación de CC-0 cuando no se visualiza la siembra peritoneal dentro del campo operatorio. CC-1 indica nódulos peritoneales $<2,5 \mathrm{~mm}$ que persisten después de la citorreducción. CC-2 indica nódulos entre $2,5 \mathrm{~mm}$ y $2,5 \mathrm{~cm}$, mientras que la puntuación CC-3 indica nódulos $>2,5 \mathrm{~cm}$ en cualquier sitio dentro del abdomen o la pelvis. Aunque tanto el CC-0 como el CC-1 corresponden con una citorreducción completa, en la práctica clínica el CC-1 sólo se considera una medida apropiada de la eficacia del HIPEC en la CPCCR, mientras que el CC-0 se considera necesario antes de que se puedan alcanzar los máximos índices de sobrevida; CC-2 y CC-3 se designan como citorreducciones incompletas. ${ }^{(43)}$

El éxito del CRS y un bajo valor de ICP son los principales indicadores de pronóstico para la sobrevida. Para los pacientes con una puntuación ICP alta, la decisión de proceder a la citorreducción debe considerarse según el estado de salud general del paciente, la biología del tumor y la capacidad de lograr una resección CC-0. ${ }^{(22)}$

\section{QUIMIOTERAPIA INTRAPERITONEAL}

Es un tipo de quimioterapia intrarregional, la cual pretende lograr altas concentraciones de un fármaco citotóxico en tumores localizados en la cavidad peritoneal. Las moléculas más utilizadas son el 5-FU, mitomicina $\mathrm{C}$, doxorubicina, cisplatino, paclitaxel, gemcitabina, etopósido, bleomicina, metotrexato, melfalán, interferón, oxaliplatino e irinotecán, las cuales pueden ser utilizadas de manera aislada o en combinación cuando se realiza justo después de la cirugía y antes de la aparición de adherencias quirúrgicas. 
Las concentraciones intraperitoneales frente a las concentraciones plasmáticas de los diferentes agentes de quimioterapia varían de 18 a 20 veces, reportándose una mayor concentración intraperitoneal; en algunos casos, desde 120-1 000 veces mayor para otros taxanos y paclitaxel respectivamente. ${ }^{(21)}$ Breton et al (1) concluyeron que las concentraciones tisulares con la quimioterapia intraperitoneal puede variar inclusive entre 20 a 400 veces más, a diferencia de las administradas por vía endovenosa.

Existe una modalidad denominada quimioterapia intraperitoneal posoperatoria precoz, la cual se inicia luego de la extirpación tumoral, entre los días 0 y 5 del postoperatorio inmediato. La solución acumulada durante 24 horas se retira diariamente mediante catéteres. Como desventaja, pueden presentarse mayores efectos adversos como: infecciones, obstrucción intestinal y las propias complicaciones del uso de catéteres intraabdominales. ${ }^{(44)}$

\section{QUIMIOTERAPIA INTRAPERITONEAL HIPERTÉRMICA}

Existen algunos medicamentos que asociados con calor ocasionan un shock tóxico directo sobre las células tumoralesEsta combinación de tratamiento regional recibe el nombre de HIPEC. ${ }^{(45)}$ Esta ha demostrado eficacia clínica antitumoral por un mecanismo directo, como consecuencia de una mayor termosensibilidad de las células tumorales o como efecto potenciador de la radioterapia y quimioterapia. Esto es producto del contacto local del fármaco intraperitoneal con el tejido tumoral, lo cual dependerá a su vez de la capacidad de penetración, tamaño, permeabilidad del fármaco, tiempo de contacto con el tejido y potencialización dada por la hipertermia. ${ }^{(1)}$ Se han realizado estudios en animales que evidencian mayor beneficio terapéutico de HIPEC con relación a la hipertermia y quimioterapia intraperitoneal como tratamientos individuales. In vitro, las células tumorales se destruyen cuando se alcanza una temperatura de $43^{\circ} \mathrm{C}$, en cambio, las células sanas resisten temperaturas de hasta $45 \circ \mathrm{C}$. Los fármacos penetran mejor cuando se alcanzan temperaturas superiores a $39-42^{\circ} \mathrm{C}$, el calor produce un descenso de la presión intersticial y esto hace que los fármacos puedan atravesar mejor los tejidos, producen efectos en el citoesqueleto, altera funciones propias de la célula como el transporte intercelular, potencial de membrana y apoptosis celular. De igual modo, hay alteración en el ADN celular, en las proteínas e incluso inducción de la síntesis de proteínas de choque térmico (HSP del inglés, "heat shock proteins"), las cuales desempeñan importantes funciones en la protección celular contra diversos tipos de estrés como: la isquemia, el estrés por calor y el estrés oxidativo. ${ }^{(44)}$ Pelz et $a l^{(46)}$ realizaron un estudio que tenía como objetivo investigar los mecanismos moleculares de las HSP junto a los efectos de HIPEC; ellos demostraron que a $41^{\circ} \mathrm{C}$, la tasa de proliferación celular se reduce al mínimo $y$, sinérgicamente, la cantidad de células apoptóticas se incrementa al máximo. Por el contrario, la sobreexpresión de la HSP se observó a $43^{\circ} \mathrm{C}$, evidenciándose una disminución de la apoptosis y el aumento de las tasas de proliferación en comparación con los efectos a 41 으. Esto prueba que la respuesta de la célula tumoral depende del choque térmico, lo que podría afectar los efectos antiproliferativos, apoptóticos deseados y podrían inducir mecanismos de protección de las células tumorales. El efecto citotóxico de la hipertermia correlacionado con los citostáticos se produce desde $39 \circ \mathrm{C}$ a $42-43 \circ \mathrm{C}$ ya que por encima de $43^{\circ} \mathrm{C}$ se puede alterar la permeabilidad y la viabilidad del intestino delgado, el cual es un factor limitante. ${ }^{(30)}$

La mitomicina-C fue el primer agente implementado de manera intraperitoneal y hoy día lo siguen empleando(22,30); es un antibiótico antitumoral con grandes ventajas farmacocinéticas ya que su concentración en la cavidad peritoneal es prolongada. Por otro lado, el oxaliplatino al administrarlo con calor alcanza concentraciones mayores en peritoneo que en plasma. ${ }^{(30)}$ Los esquemas que se utilizan en la actualidad son: (22)

- Mitomicina $30 \mathrm{mg}$ en el tiempo 0, seguido de mitomicina $10 \mathrm{mg}$ comenzando a los $60 \mathrm{~min}$ y continuando por $90 \mathrm{~min}$.

- Mitomicina $30 \mathrm{mg} / \mathrm{m}^{2}$ durante 90-110 min.

- Oxaliplatino $300 \mathrm{mg} / \mathrm{m}^{2}$ durante 30 minutos para pacientes con ICP de 11-15.

Se describen a continuación dos técnicas para la aplicación de HIPEC, la técnica abierta o coliseum descrita por Sugarbaker consiste en aplicar HIPEC con el abdomen abierto, luego de la eliminación completa de la enfermedad tumoral peritoneal, se administra los medicamentos de quimioterapia por toda la cavidad abdominal con la temperatura adecuada; luego se aspira el medicamento y se realizan las suturas digestivas. En cambio, la técnica cerrada, aplica HIPEC a vientre cerrado con la finalidad de incrementar la penetración del medicamento mediante una mayor presión generada por volúmenes de solución superior a los utilizados en la técnica abierta. ${ }^{(44)}$

Desde su difusión local, la CP puede beneficiarse de tratamientos con un intento curativo en casos seleccionados. 
En los últimos años, el tratamiento con CRS asociada a HIPEC es una técnica terapéutica agresiva con una tasa de sobrevida a 5 años que va desde $30 \%$ a $48 \% .{ }^{(47)}$ No obstante, la técnica no se ha popularizado en todo el mundo debido a que es un procedimiento complejo con resecciones extensas, multiviscerales, frecuentemente con más de dos anastomosis, quimioterapia en altas concentraciones, tiempos quirúrgicos muy largos (en ocasiones hasta 10 a 14 horas); además, el personal de salud involucrado requiere especialización en la técnica. Se desconoce de los costos locales porque nunca se ha realizado en Panamá.

\section{LA CIRUGÍA LAPAROSCÓPICA CRS MÁS HIPEC}

El interés en un enfoque laparoscópico de la cirugía oncológica ha aumentado durante la última década. Las preocupaciones anteriores incluían la hipótesis de un mayor riesgo de diseminación intraperitoneal de las células cancerosas durante la laparoscopia contra la técnica convencional. Este enfoque mínimamente invasivo es factible y seguro en un grupo muy seleccionado de pacientes con neoplasias malignas de la superficie del peritoneo, que incluye pacientes con tumores de bajo grado con diseminación peritoneal limitada, definida como una ICP de 10 o menos. Dentro de las indicaciones más comunes se incluyen: la paliación de la ascitis intratable, paliación de la obstrucción intestinal, evaluación de respuestas a la quimioterapia neoadyuvante en pacientes con carcinomatosis establecida de alto grado, y como una herramienta de puesta en escena para determinar si los pacientes son candidatos para CRS más HIPEC. Varios estudios han demostrado una mayor penetración de los fármacos en los tejidos con la técnica laparoscópica HIPEC, apoyando la hipótesis sobre el papel del aumento de la presión intraabdominal inducida por el neumoperitoneo en la farmacocinética de las drogas, con una absorción más rápida y una mayor penetración de los agentes quimioterapéuticos. ${ }^{(43)}$

Aún existen informes limitados sobre la posibilidad de combinar la cirugía laparoscópica CRS con el HIPEC en humanos, pero debe reservarse para pacientes con enfermedad no agresiva y extensión limitada. Algunos grupos quirúrgicos citorreductores experimentados han informado de una eficacia y seguridad similares utilizando esta técnica cuando se compara con el enfoque abierto con una gran incisión en la línea media, la cirugía laparoscópica disminuye la morbilidad de la herida, la duración de la estancia hospitalaria y permite una pronta recuperación. ${ }^{(48)}$
Dentro de las limitaciones se encuentra la capacidad de realizar una exploración exhaustiva de todo el abdomen y la pelvis para determinar un verdadero ICP. Podría ser difícil de explorar áreas como: la raíz del intestino delgado, el mesenterio o el peritoneo diafragmático detrás del hígado y el bazo. Para los pacientes con tumores de bajo grado, CRS limitada y laparoscópica previa, las recurrencias pueden detectarse con un seguimiento regular y podría ser tratado con un procedimiento tanto abierto como con un enfoque laparoscópico. ${ }^{(49)}$

\section{PRONÓSTICO LUEGO DE LA INTERVENCIÓN}

La mediana de sobrevida general para la CPCCR fue de 34 meses para el CRS/HIPEC con una sobrevida de cinco años pronosticada en un $40 \% .{ }^{(28)}$ En un estudio de Halkia et al. ${ }^{(50)}$ compararon el porcentaje de morbimortalidad de ambas técnicas y resultó que la morbilidad luego de realizar una HIPEC abierta era de $55 \%$ y $40 \%$ luego de una HIPEC cerrada, de igual modo la mortalidad para HIPEC abierta era de $3.3 \%$ y de $0 \%$ luego de una HIPEC cerrada, demostrando un grado de beneficio a favor de realizar la técnica cerrada. Por otra parte Rodríguez Silva y colaboradores en España en el 2017, concluyeron que al optar por la técnica cerrada se lograba mejores condiciones intraoperatorias, temperatura y mayor estabilidad hemodinámica. ${ }^{(51)}$

Como toda intervención quirúrgica, los pacientes están expuestos a múltiples complicaciones. Las causas más comunes de muerte incluyen: fuga anastomótica, sepsis y hemorragia postoperatoria. ${ }^{(52)}$ Breton et al (1) expresa que la morbimortalidad, en su mayoría es secundaria a complicaciones posquirúrgicas, supresión medular e insuficiencia respiratoria, siendo responsables del $10 \%$ de las reintervenciones quirúrgicas. La amplia exposición anatómica y duración de la intervención, aunado al componente inmunosupresor de la quimioterapia preoperatoria e intraoperatoria podrían dilucidar este desenlace. La CRS/HIPEC, se ha relacionado con una incidencia de dehiscencia anastomótica superior a la cirugía colorrectal estándar, atribuida a alteraciones del proceso de cicatrización por los citostáticos intraperitoneales, especialmente la mitomicina $\mathrm{C}$ y el cisplatino. Entre otras variables que influyen en la morbimortabilidad de dicho procedimiento se encuentran: tiempo quirúrgico prolongado, estado nutricional preoperatorio, requerimiento de transfusión o la combinación de agentes sistémicos; sin embargo, estudios actuales no confirman estos datos. $^{(30)}$ 
Según un análisis de la base de datos del Programa Nacional de Mejora de la Calidad Quirúrgica de los Estados Unidos, los pacientes que se someten a la CRS/HIPEC tienen una estancia hospitalaria media de 13 días, una tasa de readmisión del 11\%, con una tasa de morbilidad y mortalidad del $33 \%$ y $2 \%$ respectivamente. ${ }^{(52)}$ Aunque los resultados con la implementación de CRS/HIPEC en el manejo de la CPCCR son favorables, muchos pacientes sufren recurrencias de la enfermedad después de este tratamiento. ${ }^{(53)}$ Un estudio que incluyó 414 pacientes evaluó de forma retrospectiva a todos los pacientes que se sometieron a CRS/HIPEC por CPCCR entre 2004 y 2015, y registró que 106 de estos pacientes (25.6\%) fueron diagnosticados con recurrencia peritoneal aislada de la enfermedad después de CRS/HIPEC, de los cuales 43 (40.6\%) fueron tratados con intención curativa y 63 (59.4\%) se sometieron a tratamiento con propósito paliativo. Por lo que se concluyó que, aproximadamente uno de cada cuatro pacientes desarrollará recurrencias peritoneales aisladas. ${ }^{(54)}$

Por el contrario, en el estudio Braam et al ${ }^{(53)}$ evidenciaron que de una muestra de 287 pacientes con CP que fueron tratados con CRS completa (RO/1) e HIPEC a quienes se les siguió por una mediana de tiempo de 26,6 meses, 97 pacientes (73\%) sufrieron recurrencia peritoneal aislada; teniendo en cuenta que, el intervalo de tiempo entre la terapia HIPEC y la recurrencia fue de 11.4 meses. Inclusive un grupo de investigadores en los Países Bajos lograron constatar mediante la realización de un análisis multivariante que la aparición de complicaciones postoperatorias que requieren una intervención mayor o igual a 3 después de CRS/HIPEC, fue el único factor de riesgo significativo encontrado para la recurrencia temprana (OR 2.3; $p=0.046)$. En este estudio 46 de 133 pacientes (35\%) desarrollaron recurrencia en 12 meses con una mediana de sobrevida de 19.3 meses. ${ }^{(55)}$

\section{REALIDAD EN PANAMÁ}

Según los últimos datos de la Organización Mundial de la Salud (OMS), en el año 2018 Panamá contó con un total de 781 casos nuevos de CCR, de los cuales 359 fueron mujeres y 422 varones. En Panamá, el número de muertes registradas por CCR en el año 2018 fue de 374 (es decir 2,13\% de todas las muertes); además la tasa de mortalidad por edad es de 9.86 por 100000 de población. De hecho, más del $90 \%$ de los casos que se presentan son personas mayores de 50 años y el $80 \%$ de los pacientes diagnosticados con esta enfermedad, no cuentan con un historial familiar relacionado a este tumor. El CCR ocupa el segundo lugar en el listado de tipos de cáncer más común en la población; por lo que podemos concluir que la elevada incidencia y mortalidad del CCR hacen de este un problema de salud pública en Panamá. ${ }^{(56)}$

Debido al sesgo de publicaciones referentes a la $C P$, se desconoce mucho sobre su situación actual, así como la mortalidad de la misma en Panamá, ya que hasta la fecha únicamente contamos con el registro hospitalario de cáncer del año 2016 por parte del Instituto Oncológico Nacional (ION), donde se registró una incidencia del $0.2 \%$ del total de cánceres registrados, lo que equivalió a un total de 7 pacientes de los cuales 5 eran del sexo masculino y 2 eran del sexo femenino lo que representó un $0.4 \%$ y $0.1 \%$ respectivamente. La problemática de este registro radica en que no solamente se tomó en consideración a la CP sino también a los tumores malignos del retroperitoneo, haciendo menos precisa la incidencia de la misma. ${ }^{(57)}$ Sumado a esto, el inconveniente de que la gran mayoría de pacientes del ION son registrados únicamente bajo la categoría de su tumor primario, pero no de su metástasis, conlleva al enorme subregistro que hoy en día poseemos. De igual forma desconocemos la incidencia de la carcinomatosis peritoneal por subtipo tumoral. En Panamá, a pesar de contar con el personal especializado entrenado en la técnica (cirujanos oncólogos), aún no se tiene el equipo necesario y logística institucional para el HIPEC.

\section{DISCUSIÓN}

La CP sigue siendo un desafío significativo tanto diagnóstico como terapéutico para los oncólogos y cirujanos. Utilizando los datos disponibles basados en la evidencia y considerando la calidad de los estudios, podemos señalar las características clínico-patológicas de la CPCCR completamente resecado y evaluar los patrones de recurrencia, ya que la correcta identificación de estos factores puede ser útil al momento de planificar estrategias clínicas para los pacientes con $\mathrm{CP}{ }^{\left({ }^{(58)}\right.}$ Además, los avances de la biología tumoral, la clasificación molecular, la identificación de alteraciones genéticas, así como una mejor comprensión del pronóstico y de los factores de riesgo para el desarrollo de la CPCCR hacen posible perfeccionar los criterios de selección de los pacientes para este tratamiento combinado y para definir nuevos enfoques basados en actitudes proactivas.

Se ha visto que el pronóstico de los pacientes con CPCCR ha cambiado significativamente en los últimos años, así como la calidad de vida gracias a la introducción de un nuevo enfoque 
quirúrgico que combina la CRS/HIPEC y que cada vez resulta en una mayor aceptación por parte de la comunidad oncológica como una opción de tratamiento para los pacientes con CPCCR, siendo esta manifestación clínica de pronóstico variable. ${ }^{(25,59)} \mathrm{Es}$ vital tomar en cuenta que la selección precisa de pacientes ha demostrado ser un predictor clave del resultado de CRS/HIPEC.

A pesar de que años atrás eran menos los criterios que se tomaban en cuenta para seleccionar a los pacientes que recibieron este tratamiento, existía una diferencia considerable de la morbimortalidad con relación a los pacientes que no se trataban. Hoy en día, existen criterios más exhaustivos para elegir aquellos pacientes que se puedan someter a este procedimiento con menos riesgo perioperatorio y mayor sobrevida a largo plazo. No obstante, aunque el CRS/HIPEC impacte en la calidad de vida de los pacientes, el mismo no tiene efecto en el alivio de otros síntomas sistémicos que dificultan significativamente la calidad de vida restante. Además, cabe recalcar que la terapia molecularmente dirigida podría servir como vía de futuras investigaciones. ${ }^{\left({ }^{60}\right)}$ Debido al intento de unificar conceptos referentes a cuál de las diferentes modalidades del manejo de la CPCCR debe preferirse, se han comparado en diversos estudios cada una de ellas para así facilitar su elección. Con el inconveniente de la presencia de sesgo de selección en estudios basado en la población, la falta de datos sobre factores pronósticos importantes como: el estado funcional, la extensión de la enfermedad peritoneal y las comorbilidades, los cuales no son registrados muchas veces, limita la capacidad para poder concluir el efecto de estas nuevas técnicas terapéuticas.

Pese a la existencia de múltiples estudios que avalan la utilización en conjunto de CRS e HIPEC en el manejo de la CPCCR, un estudio reciente por Cashin et al ${ }^{(24)}$ asevera que dicha modalidad de tratamiento sólo se encuentra disponible en instituciones especializadas y no está indicada para la mayoría de los pacientes con CPCCR debido a su alta morbilidad y mortalidad. Siendo la tasa de grave morbilidad a los 60 días mayor con CRS/HIPEC que con CRS solo $(24.1 \%$ vs $13.6 \%$, respectivamente). En contraparte Gupta y otros ${ }^{(61)}$ también compararon la sobrevida de pacientes tratados con CRS/HIPEC cerrada y pacientes tratados únicamente con CRS. Como resultado, la sobrevida a 4 años de la CRS/HIPEC cerrada y la CRS fue de $58.9 \%$ y $33.33 \%$, respectivamente. Lo que nos lleva al cuestionamiento de las discrepancias entre la evidencia existente.
Entre las comparaciones nos encontramos con la duda de si es mejor la utilización de la CRS abierta vs laparoscópica, Passot et al. ${ }^{(48)}$ concluyeron que la CRS laparoscópica combinada con el HIPEC es factible y segura para el tratamiento curativo de pacientes estrictamente seleccionados con malignidad de la superficie del peritoneo, pudiendo reducir las complicaciones postoperatorias y la duración de la estancia en el hospital.

\section{CONCLUSIÓN}

Consecuente al avance en el conocimiento de la fisiopatogenia, comportamiento y mecanismos de diseminación, hoy en día contamos con un gran número de publicaciones referentes al tratamiento de la enfermedad metastásica peritoneal de origen colorrectal, las cuales respaldan un aumento en la tasa de sobrevida de hasta 40 meses en pacientes seleccionados. Para conseguir esta meta, se ha sugerido un abordaje multimodal basado en una citorreducción oncológica radical con HIPEC.

Es apreciable como a lo largo del tiempo se han creado y modificado herramientas que incluso antes de realizar el procedimiento quirúrgico, son fundamentales para mejorar la eficacia del mismo.

A medida que se identifiquen más pacientes aptos para este procedimiento, mayor será el número de personas que se beneficien del empleo de estas técnicas. A pesar de que actualmente muchos centros a nivel mundial cuentan con CRS/HIPEC, aún queda un porcentaje considerable de instituciones en América Latina, incluyendo Panamá, que carecen de las mismas, por lo que consideramos que la evidencia recolectada podría incentivar su instauración para el adecuado manejo de la CPCCR.

\section{AGRADECIMIENTOS}

Agradecemos al Dr. Gerardo Victoria por incentivarnos a la realización de esta revisión, además de brindarnos recomendaciones referentes al proceso de publicación de la misma.

\section{DECLARACIÓN DE CONFLICTOS DE INTERESES}

Los autores no declaran conflicto de interés. 


\section{BIBLIOGRAFÍA}

1. Breton C, Pertuz JE, Calderón D, Angulo J, Pacheco M. Manejo de carcinomatosis peritoneal con citorreducción más quimioterapia hipertérmica intraoperatoria (HIPEC). Revista Repertorio de Medicina y Cirugía. 2020 Jan 30;29(1).

2. Sánchez E, Espin F. Carcinomatosis peritoneal. Revisión de conjunto. Revista Médica de Trujillo. 2019 Apr 2;14(1).

3. Grass F, Vuagniaux A, Teixeira-Farinha H, Lehmann K, Demartines $N$, Hübner $M$. Systematic review of pressurized intraperitoneal aerosol chemotherapy for the treatment of advanced peritoneal carcinomatosis. British Journal of Surgery. 2017 May;104(6):669-78.

4. Kok N, de Hingh I. Cytoreductive surgery and hyperthermic intraperitoneal chemotherapy for peritoneal metastases of colorectal origin. British Journal of Surgery. 2017;104(4):313-315.

5. Ministerio de Salud de Panamá. Registro Nacional de Cáncer de Panamá. (2012). 6. González, D, Ruso Martínez, L, Telles Onetti, L Carcinomatosis peritoneal : conceptos y tratamiento con cirugía y quimioterapia hipertérmica intraperitoneal. [Internet]. Montevideo, Uruguay: Universidad de la República ; 2014. [citado: 2020, mayo] 110 p.

7. Graniel-Palafox LE, Guerrero-Avendaño G. Vías de diseminación y sitios frecuentes de implantación metastásica en carcinomatosis peritoneal; hallazgos por tomografía. Anales de Radiología, Mexico. 2013 Jan 1;12(1).

8. McMullen JR, Selleck M, Wall NR, Senthil M. Peritoneal carcinomatosis: limits of diagnosis and the case for liquid biopsy. Oncotarget. 2017 Jun 27;8(26):43481.

9. Calderón Á, Cuéllar D. PET/CT 18F-FDG en carcinomatosis peritoneal: revisión de la fisiopatología y presentación de dos casos. Revista Colombiana de Cancerología. 2018 Jun;22(2):92-5.

10. Franiel T, Diederichs G, Engelken F, Elgeti T, Rost J, Rogalla P. Multi-detector CT in peritoneal carcinomatosis: diagnostic role of thin slices and multiplanar reconstructions. Abdominal imaging. 2009 Jan 1;34(1):49-54.

11. Coccolini F, Gheza F, Lotti M, Virzì S, lusco D, Ghermandi C, Melotti R, Baiocchi G, Giulini SM, Ansaloni L,
Catena F. Peritoneal carcinomatosis. World journal of gastroenterology: WJG. 2013 Nov 7;19(41):6979.

12. Grotz TE, Fournier KF, Mansfield PF. Patient selection for cytoreductive surgery. Surgical Oncology Clinics. 2018 Jul 1;27(3):443-62.

13. Baratti D, Kusamura S, Azmi N, Guaglio M, Montenovo M, Deraco M. Colorectal Peritoneal Metastases Treated by Perioperative Systemic Chemotherapy and Cytoreductive Surgery With or Without Mitomycin C-Based HIPEC: A Comparative Study Using the Peritoneal Surface Disease Severity Score (PSDSS). Annals of surgical oncology. 2020 Jan;27(1):98-106.

14. Klaver YL, Chua TC, de Hingh IH, Morris DL. Outcomes of elderly patients undergoing cytoreductive surgery and perioperative intraperitoneal chemotherapy for colorectal cancer peritoneal carcinomatosis. Journal of surgical oncology. 2012 Feb 1;105(2):113-8.

15. Razenberg LG, Van Gestel YR, Creemers GJ, Verwaal VJ, Lemmens VE, de Hingh IH. Trends in cytoreductive surgery and hyperthermic intraperitoneal chemotherapy for the treatment of synchronous peritoneal carcinomatosis of colorectal origin in the Netherlands. European Journal of Surgical Oncology (EJSO). 2015 Apr 1;41(4):466-71.

16. Esquivel J, Sticca R, Sugarbaker P, Levine E, Yan TD, Alexander R, Baratti D, Bartlett D, Barone R, Barrios P, Bieligk S. Cytoreductive surgery and hyperthermic intraperitoneal chemotherapy in the management of peritoneal surface malignancies of colonic origin: a consensus statement. Annals of surgical oncology. 2007 Jan 1;14(1):128-33.

17. Elias D, Gilly F, Boutitie F, Quenet F, Bereder JM, Mansvelt B, Lorimier G, Dube P, Glehen O. Peritoneal colorectal carcinomatosis treated with surgery and perioperative intraperitoneal chemotherapy: retrospective analysis of 523 patients from a multicentric French study. J Clin Oncol. 2010 Jan $1 ; 28(1): 63-8$.

18. Kuijpers AM, Mirck B, Aalbers AG, Nienhuijs SW, de Hingh IH, Wiezer MJ, van Ramshorst B, van Ginkel RJ, Havenga $\mathrm{K}$, Bremers AJ, de Wilt JH. Cytoreduction and HIPEC in the Netherlands: nationwide long-term outcome following the Dutch protocol. Annals of surgical oncology. 2013 Dec 1;20(13):4224-30. 
19. Flicek K, Ashfaq A, Johnson CD, Menias C, Bagaria S, Wasif $\mathrm{N}$. Correlation of radiologic with surgical peritoneal cancer index scores in patients with pseudomyxoma peritonei and peritoneal carcinomatosis: how well can we predict resectability?. Journal of Gastrointestinal Surgery. 2016 Feb $1 ; 20(2): 307-12$.

20. Goéré D, Souadka A, Faron M, Cloutier AS, Viana B, Honoré C, Dumont F, Elias D. Extent of colorectal peritoneal carcinomatosis: attempt to define a threshold above which HIPEC does not offer survival benefit: a comparative study. Annals of surgical oncology. 2015 Sep 1;22(9):2958-64.

21. López-Basave HN, Morales-Vásquez F, Luna Ortiz K, Méndez Herrera C, Ruiz-Molina JM. Citorreducción e HIPEC en carcinomatosis peritoneal. Experiencia del Instituto Nacional de Cancerología de México. Cirujano general. 2014;36(3):138-44.

22. The Chicago Consensus on Peritoneal Surface Malignancies: standards. Annals of surgical oncology.2020; 126(11), 2534-2540. 23. Winer J, Zenati M, Ramalingam L, Jones H, Zureikat A, Holtzman M, Lee K, Ahrendt S, Pingpank J, Zeh HJ, Bartlett DL. Impact of aggressive histology and location of primary tumor on the efficacy of surgical therapy for peritoneal carcinomatosis of colorectal origin. Annals of surgical oncology. 2014 May 1;21(5):1456-62.

24. Cashin PH, Graf W, Nygren P, Mahteme H. Comparison of prognostic scores for patients with colorectal cancer peritoneal metastases treated with cytoreductive surgery and hyperthermic intraperitoneal chemotherapy. Annals of surgical oncology. 2013 Dec 1;20(13):4183-9.

25. Chua TC, Morris DL, Esquivel J. Impact of the peritoneal surface disease severity score on survival in patients with colorectal cancer peritoneal carcinomatosis undergoing complete cytoreduction and hyperthermic intraperitoneal chemotherapy. Annals of Surgical Oncology. 2010 May 1;17(5):1330-6.

26. Arjona-Sánchez A, Rodríguez-Ortiz L, Baratti D, Schneider MA, Gutiérrez-Calvo A, García-Fadrique A, Tuynman JB, Cascales-Campos PA, Martín VC, Morales R, Salti GI. RAS mutation decreases overall survival after optimal cytoreductive surgery and hyperthermic intraperitoneal chemotherapy of colorectal peritoneal metastasis: a modification proposal of the peritoneal surface disease severity score. Annals of surgical oncology. 2019 Aug 15;26(8):2595-604.
27. Yong ZZ, Tan GH, Shannon N, Chia C, Teo MC. PROPSA novel Pre-Operative Predictive Score for unresectability in patients with colorectal peritoneal metastases being considered for cytoreductive surgery (CRS) and hyperthermic intraperitoneal chemotherapy (HIPEC). World journal of surgical oncology. 2019 Dec 1;17(1):138.

28. Cashin PH, Graf W, Nygren P, Mahteme $H$. Cytoreductive surgery and intraperitoneal chemotherapy for colorectal peritoneal carcinomatosis: prognosis and treatment of recurrences in a cohort study. European Journal of Surgical Oncology (EJSO). 2012 Jun 1;38(6):509-15.

29. Nikolic S, Dzodic R, Zegarac M, Djurisic I, Gavrilovic D, Vojinovic V, Kocic M, Santrac N, Radlovic P, Radosavljevic D, Pupic G. Survival prognostic factors in patients with colorectal peritoneal carcinomatosis treated with cytoreductive surgery and intraoperative hyperthermic intraperitoneal chemotherapy: a single institution experience. J buon. 2014 Jan $1 ; 19(1): 66-74$

30. Morales Soriano R. Estudio de los factores asociados con la morbi-mortalidad postoperatoria y la supervivencia tras citorreducción quirúrgica y quimioterapia intraperitoneal hipertérmica (HIPEC), en pacientes con carcinomatosis peritoneal por cáncer de colon. España. Universitat de les Illes Balears. 2019.

31. Waite $\mathrm{K}$, Youssef $\mathrm{H}$. The role of neoadjuvant and adjuvant systemic chemotherapy with cytoreductive surgery and heated intraperitoneal chemotherapy for colorectal peritoneal metastases: a systematic review. Annals of surgical oncology. 2017 Mar 1;24(3):705-20.

32. Huang $\mathrm{C}$, Yang $\mathrm{X}, \mathrm{Yu} \mathrm{Y}, \mathrm{Wu} \mathrm{H}$, Liu $\mathrm{Y}$, Yonemura $\mathrm{Y}$ et al. Cytoreductive Surgery plus Hyperthermic Intraperitoneal Chemotherapy Improves Survival for Patients with Peritoneal Carcinomatosis from Colorectal Cancer: A Phase II Study from a Chinese Center. PLoS ONE. 2014;9(9):e108509.

33. Chua TC, Morris DL, Saxena A, Esquivel J, Liauw W, Doerfer J, Germer CT, Kerscher AG, Pelz JO. Influence of modern systemic therapies as adjunct to cytoreduction and perioperative intraperitoneal chemotherapy for patients with colorectal peritoneal carcinomatosis: a multicenter study. Annals of surgical oncology. 2011 Jun 1;18(6):1560-7

34. Van Eden W, Kok N, Jóźwiak K, Lahaye M, Beets G, van Leerdam $\mathrm{M}$ et al. Timing of Systemic Chemotherapy in Patients 
With Colorectal Peritoneal Carcinomatosis Treated With Cytoreductive Surgery and Hyperthermic Intraperitoneal Chemotherapy. Diseases of the Colon \& Rectum. 2017;60(5):477-487.

35. Sánchez-Hidalgo JM, Rodríguez-Ortiz L, ArjonaSánchez Á, Rufián-Peña S, Casado-Adam Á, Cosano-Álvarez A, Briceño-Delgado J. Colorectal peritoneal metastases: Optimal management review. World journal of gastroenterology. 2019 Jul 21;25(27):3484.

36. Van Dijk T, Tamas K, Beukema J, Beets G, Gelderblom $A$, de Jong $K$ et al. Evaluation of short-course radiotherapy followed by neoadjuvant bevacizumab, capecitabine, and oxaliplatin and subsequent radical surgical treatment in primary stage IV rectal cancer. Annals of Oncology. 2013;24(7):17621769.

37. Leimkühler $\mathrm{M}$, Hemmer $\mathrm{PH}$, Reyners $\mathrm{AK}$, de Groot DJ, van Ginkel RJ, Been LB, de Bock GH, van Leeuwen BL. Neoadjuvant chemotherapy followed by cytoreductive surgery and hyperthermic intraperitoneal chemotherapy for colorectal cancer: a feasibility and safety study. World journal of surgical oncology. 2019 Dec 1;17(1):14.

38. Passot G, You B, Boschetti G, Fontaine J, Isaac S, Decullier E, Maurice C, Vaudoyer D, Gilly FN, Cotte E, Glehen O. Pathological response to neoadjuvant chemotherapy: a new prognosis tool for the curative management of peritoneal colorectal carcinomatosis. Annals of surgical oncology. 2014 Aug 1;21(8):2608-14.

39. Beal EW, Suarez-Kelly LP, Kimbrough CW, Johnston FM, Greer J, Abbott DE, Pokrzywa C, Raoof M, Lee B, Grotz TE, Leiting JL. Impact of Neoadjuvant Chemotherapy on the Outcomes of Cytoreductive Surgery and Hyperthermic Intraperitoneal Chemotherapy for Colorectal Peritoneal Metastases: A Multi-Institutional Retrospective Review. Journal of Clinical Medicine. 2020 Mar;9(3):748.

40. Mirnezami R, Moran BJ, Harvey K, Cecil T, Chandrakumaran K, Carr N, Mohamed F, Mirnezami AH. Cytoreductive surgery and intraperitoneal chemotherapy for colorectal peritoneal metastases. World Journal of Gastroenterology WJG. 2014 Oct 14;20(38):14018.

41. Losa F, Barrios P, Salazar R, Torres-Melero J, Benavides M, Massuti T, Ramos I, Aranda E. Cytoreductive surgery and intraperitoneal chemotherapy for treatment of peritoneal carcinomatosis from colorectal origin. Clinical and Translational Oncology. 2014 Feb 1;16(2):128-40.

42. Granados García M, Arrieta Rodríguez O, de León C. Oncología y cirugía: bases y principios. 1. a ed. México: Editorial El Manual Moderno; 2013. Capítulo 40 Carcinomatosis Peritoneal; p. 447

43. Arjona-Sanchez A, Esquivel J, Glehen O, Passot G, Turaga KK, Labow D, Rufian-Peña S, Morales R, van der Speeten $K$. A minimally invasive approach for peritonectomy procedures and hyperthermic intraperitoneal chemotherapy (HIPEC) in limited peritoneal carcinomatosis: The American Society of Peritoneal Surface Malignancies (ASPSM) multi-institution analysis. Surgical endoscopy. 2019 Mar 15;33(3):854-60.

44. Villaverde RM, Calvo AG, Soto MÁ. Protocolo de manejo de la carcinomatosis peritoneal. Medicine-Programa de Formación Médica Continuada Acreditado. 2017 May 1;12(32):1941-4.

45. Chia, C. S., You, B., Decullier, E., Vaudoyer, D., Lorimier, G., Abboud, K., \& BIG RENAPE Group. (2016). Patients with peritoneal carcinomatosis from gastric cancer treated with cytoreductive surgery and hyperthermic intraperitoneal chemotherapy: is cure a possibility?. Annals of surgical oncology, 23(6), 1971-1979.

46. Pelz JO, Vetterlein M, Grimmig T, Kerscher AG, Moll E, Lazariotou M, Matthes N, Faber M, Germer CT, Waaga-Gasser AM, Gasser M. Hyperthermic intraperitoneal chemotherapy in patients with peritoneal carcinomatosis: role of heat shock proteins and dissecting effects of hyperthermia. Annals of surgical oncology. 2013 Apr 1;20(4):1105-13.

47. Auricchio A, Cardella F, Mabilia A, Basile N, Erario S, Del Sorbo G, Castellano P, Galizia G, Lieto E. Peritoneal Carcinoatosis from Colorectal Cancer: Fluorescence-Guided Surgery with Indocyanine Green.

48. Passot G, Bakrin N, Isaac S, Decullier E, Gilly FN, Glehen O, Cotte E. Postoperative outcomes of laparoscopic vs open cytoreductive surgery plus hyperthermic intraperitoneal chemotherapy for treatment of peritoneal surface malignancies. European Journal of Surgical Oncology (EJSO). 2014 Aug 1;40(8):957-62.

49. Arjona-Sanchez A, Rufian-Peña S, Sanchez-Hidalgo JM, Casado-Adam A, Cosano-Alvarez A, Briceño-Delgado J. 
Cytoreductive surgery and intraperitoneal hyperthermic chemotherapy (HIPEC) by minimally invasive approach, an initial experience. World journal of surgery. 2018 Oct $1 ; 42(10): 3120-4$.

50. Halkia E, Tsochrinis A, Vassiliadou D, Pavlakou A, Vaxevanidou A, Datsis $A$ et al. Peritoneal Carcinomatosis: Intraoperative Parameters in Open (Coliseum) versus Closed Abdomen Hipec. International Journal of Surgical Oncology. 2015;2015:1-6.

51. Rodríguez Silva C, Moreno Ruiz F, Bellido Estévez I, Carrasco Campos J, Titos García A, Ruiz López M et al. Are there intra-operative hemodynamic differences between the Coliseum and closed HIPEC techniques in the treatment of peritoneal metastasis? A retrospective cohort study. World Journal of Surgical Oncology. 2017;15(1).

52. Jafari M, Halabi W, Stamos M, Nguyen V, Carmichael J, Mills S et al. Surgical Outcomes of Hyperthermic Intraperitoneal

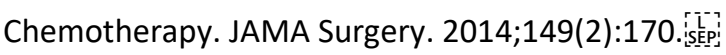

53. Braam $H$, van Oudheusden $T$, de Hingh I, Nienhuijs $S$, Boerma D, Wiezer $\mathrm{M}$ et al. Patterns of recurrence following complete cytoreductive surgery and hyperthermic intraperitoneal chemotherapy in patients with peritoneal carcinomatosis of colorectal cancer. Journal of Surgical Oncology. 2014;109(8):841-847.

54. Van Eden W, Elekonawo F, Starremans B, Kok N, Bremers A, de Wilt J et al. Treatment of Isolated Peritoneal Recurrences in Patients with Colorectal Peritoneal Metastases Previously Treated with Cytoreductive Surgery and Hyperthermic Intraperitoneal Chemotherapy. Annals of Surgical Oncology. 2018;25(7):1992-2001

55. Simkens $G$, van Oudheusden T, Luyer M, Nienhuijs $S$, Nieuwenhuijzen $\mathrm{G}$, Rutten $\mathrm{H}$ et al. Serious Postoperative Complications Affect Early Recurrence After Cytoreductive Surgery and HIPEC for Colorectal Peritoneal Carcinomatosis. Annals of Surgical Oncology. 2014;22(8):2656-2662.

56. Información adaptada de la Sociedad Americana contra el Cáncer, del Registro Nacional de Tumores de la OMS. Globocan 2018 y de Roche, 2019.

57. Registro hospitalario de Cáncer del Instituto Oncológico Nacional, Panamá 2016.
58. Matsuda K, Hotta T, Takifuji K, Yamamoto M, Nasu T, Togo $\mathrm{N}$ et al. Clinical impact of a macroscopically complete resection of colorectal cancer with peritoneal carcinomatosis. Surgery. 2012;151(2):238-244.

59. Enblad M, Ghanipour L, Cashin PH. Prognostic scores for colorectal cancer with peritoneal metastases treated with cytoreductive surgery and hyperthermic intraperitoneal chemotherapy. International Journal of Hyperthermia. 2018 Nov 17;34(8):1390-5.

60. Aoyagi T, Terracina KP, Raza A, Takabe K. Current treatment options for colon cancer peritoneal carcinomatosis. World journal of gastroenterology: WJG. 2014 Sep 21;20(35):12493

61. Gupta N, Asif S, Gandhi J, Rajpurohit S, Singh S. Role of CRS and HIPEC in appendiceal and colorectal malignancies: Indian experience. Indian Journal of Gastroenterology. 2017 Mar 1;36(2):126-30. 